\title{
Detección precoz de trastornos del neurodesarrollo en los primeros años de vida en niños con cardiopatías congénitas
}

\author{
Early detection of neurodevelopment disorders in the first years of life \\ in children with congenital heart diseases
}

\section{Resumen}

Este estudio se ha realizado para conocer las morbilidades existentes en el desarrollo de los niños que presentan cardiopatías congénitas (CC) intervenidos quirúrgicamente mediante circulación extracorpórea (CEC) y diseñar directrices para su seguimiento e intervención temprana. Se realizó un estudio de corte cuantitativo ( $\mathrm{N}=30$ ), en base a los siguientes criterios de inclusión: edad, tipo de CC e intervención quirúrgica mediante CEC, situados en un rango de edad de I I a 42 meses. Se evaluó el desarrollo motor, cognitivo y del lenguaje a través de la Escala de Desarrollo Infantil Bayley-III y se tuvieron en cuenta las variables biomédicas. Entre los resultados destaca el hecho de que los participantes en el estudio presentan morbilidades en el área comunicativo-lingüística y motora, existen indicadores biomédicos (edad) en el desarrollo madurativo y necesitan participar en programas de atención temprana (AT) para normalizar su desarrollo. La conclusión es que esta población necesita protocolos interdisciplinares de detección, despistaje e intervención, inexistentes actualmente, y son objeto de recibir AT.

\section{Palabras clave}

Cardiopatía congénita, CEC, alteraciones en el desarrollo global, atención temprana.

\section{Abstract}

This study was carried out in order to report on existing morbidities in the development of children with congenital heart disease (CHD) who underwent Extracorporeal Circulation (ECC) surgery. The purpose of the study is also to design guidelines for the monitoring and early intervention of these conditions. A quantitative study ( $\mathrm{N}=30$ ), based on the following inclusion criteria: age, type of CHD and surgery by ECC, located in an age range of I I to 42 months, was made. The motor, cognitive and language development was evaluated with help of the Scales of Infant and Toddler Development Bayley-III. The results highlight that the participants present morbidities in the communicativelinguistic and the motor areas. There are biomedical indicators (age) in the maturational development. In order to normalize their development the children would need to participate in early intervention programs (EIP). The conclusion is that this population needs early intervention (EI) and interdisciplinary protocols for detection, screening and intervention that, on this time, are not available.
\end{abstract}

\section{Keywords}

Congenital heart disease, ECC, global development disorders, early intervention.

\author{
$\mathbf{M}^{\mathrm{a}}$ del Rosario Mendoza \\ Carretero \\ <mamendoz@ucm.es> \\ Universidad Complutense de Madrid
}

\section{Susana Ares Segura}

<susana.ares@salud.madrid.org>

Hospital Universitario La Paz de Madrid

\section{Belén Sáenz-Rico de Santiago \\ <bsaenzri@edu.ucm.es> \\ Universidad Complutense de Madrid}

Para citar:

Mendoza, M. R. et al. (20I7):

"Detección precoz de trastornos del neurodesarrollo en los primeros años de vida en niños con cardiopatías congénitas". Revista Española de Discapacidad, 5 (I): 99-I I I.

Doi: <https://doi.org/IO. 5569/23405 I04.05.01.06>

Fecha de recepción: I4-09-20I6

Fecha de aceptación: 06-04-20I7 


\section{Introducción}

Las cardiopatías congénitas (CC) son malformaciones del corazón o de los grandes vasos sanguíneos presentes en el feto o en el recién nacido (Villagrá, 20I4). Estas anomalías son debidas a un desarrollo incompleto o anómalo del corazón, pudiendo aparecer orificios en los tabiques que hay entre las cámaras del corazón, fugas en las válvulas o, incluso, que los vasos sanguíneos sean angostos.

Las CC son consideradas por la Organización Mundial de la Salud (20I6) como enfermedades crónicas, puesto que son patologías de larga duración y de progresión lenta, cuya curación no puede preverse (Aguirre et al., 2008). Las personas que las presentan pueden tener experiencias similares aunque existan diferencias en los síntomas y en la gravedad de la enfermedad.

Estas anomalías afectan a 8 de cada Iooo nacidos vivos y presentan la mayor tasa de mortalidad en el período neonatal. Sin embargo, cuando se incluyen defectos septales ventriculares leves y otros defectos menores la cifra asciende a 75 por cada Iooo nacidos vivos (Esquivel et al. 2013).

De todos los menores nacidos con CC, uno de cada tres necesita intervención quirúrgica durante el primer año de vida, según indica Arretz (2000). Williams et al. (20 I 2b) señalan que con este tipo de intervención se reducen las tasas de mortalidad en estos infantes pero que, a su vez, aumenta la probabilidad de que aparezcan morbilidades en el neurodesarrollo a largo plazo, según defiende Schaefer et al. (2013).

Estos avances han permitido progresar en el estudio de infantes con CC, ya que el objetivo de estas técnicas no se centra en mantenerles con vida, sino que se trata de mejorar su pronóstico con una mayor calidad de vida y estas morbilidades pueden ser causadas por factores biológicos como el defecto cardiaco en sí (aporte deficitario de oxígeno y/o glucosa al cerebro durante y después de la gestación) y por las terapias e intervenciones quirúrgicas a las que han sido sometidos (microembolias producidas durante la circulación extracorpórea, el cateterismo y déficit de oxígeno por parada cardiopulmonar).

Las cardiopatías congénitas presentes en los neonatos se han visto favorecidas gracias a la cirugía por circulación extracorpórea (CEC), "pasando de ser una rara entidad de altísimo riesgo a ser una estrategia habitual para la reparación de un buen número de cardiopatías" (Magliola et al. 2009: 4I 8). Esta técnica "permite sustituir temporalmente tanto la acción de bomba que ejerce el corazón, como la misión de intercambio gaseoso de los pulmones, gracias a un sistema mecánico de bombeo con capacidad para oxigenar la sangre”. Mientras se produce la CEC “el cerebro queda en manos de una circulación restringida y dependiente de la bomba de circulación extracorpórea para su oxigenación y nutrición" (Armele et al. 20I4: 8 I), pudiendo verse afectado.

Este proceso puede generar trastornos en el desarrollo con múltiples secuelas (Alcover et al., I999), produciendo lentitud en la organización de todas las funciones cerebrales superiores (Azcoaga, 1979).

Por ello, teniendo en cuenta los procesos quirúrgicos utilizados en las intervenciones destinadas a niños con CC, se observa que tras la cirugía los infantes presentan déficits neurológicos y lentificaciones en el desarrollo.

El estudio realizado por Dittrich et al. (2003) nos muestra que existen diferencias en el desarrollo motor entre los infantes debido a las malformaciones cardiacas de aquellos que no las presentan. Estas diferencias se evidencian en las destrezas motoras, en las habilidades personales y sociales, en el rendimiento cognitivo y en el lenguaje. Estos hallazgos pudieron verificar que el retraso surgió después de la intervención quirúrgica, siendo más evidentes los déficits neurológicos en los bebés que presentaban estas malformaciones. 
El estudio realizado por Matsuzaki et al. (2010) muestra que existen secuelas en el neurodesarrollo, caracterizado por un deterioro en el lenguaje y en las habilidades motoras visuales-espaciales. Estas secuelas pueden hacerse más evidentes cuando se necesita un mayor nivel cognitivo y mayores habilidades en las funciones ejecutivas.

Asimismo, Williams et al. (20I2a) defienden que los niños con CC tienen un alto riesgo de padecer problemas neurocognitivos, incluyendo dificultades de aprendizaje, problemas en la conducta y lentitud mental.

Apoyando lo anterior, Mulkey et al. (20I4) destacan que los educandos con CC tienen dificultades en las matemáticas, disminución en la alfabetización y un desarrollo motor inferior aunque presentan un cociente intelectual (CI) normal.

Teniendo en cuenta los estudios realizados por Gunn et al. (2OI2), los niños con CC que sobrevivieron a la intervención quirúrgica y fueron evaluados a los dos años de edad con la Escala Bayley de Desarrollo Infantil-III ( $3^{\text {a }}$ edición) (2005) mostraron que existe un retraso severo en el desarrollo neurológico tanto a nivel cognitivo como en el área comunicativalingüística o en el desarrollo motor.

A su vez, podemos destacar que existen estudios de CC vinculados con la discapacidad, donde Mulkey et al. (20I4) señalan que los niños con CC que presentan discapacidades múltiples están escolarizados en centros de educación especial.

Independientemente de la presencia o ausencia de discapacidad en las CC, Mulkey et al. (20I4) señalan que la intervención temprana puede mejorar a los cardiópatas infantiles. Junto a ellos, Williams et al. (20I2b) recomiendan y consideran que es conveniente que estos niños reciban AT, pues varios autores han encontrado en recién nacidos a término con cardiopatías congénitas, patrones de desarrollo similares a los niños prematuros (Esquivel et al. 2013). Estos últimos reciben AT (GAT, 2000) mientras que los primeros no, a pesar de que les beneficiaría, según indican Williams et al. (20I2b).

La atención temprana, dirigida a la población infantil de o-6 años, a la familia y al entorno, puede dar respuesta a las necesidades permanentes o transitorias que presentan los infantes con trastornos en su desarrollo o que tienen el riesgo de padecerlos. Siguiendo a González (20I3) la AT está dirigida a niños con déficits físicos, psíquicos o sensoriales y para aquellos que puedan presentar problemas madurativos o de adaptación, como los infantes con CC.

Por todo ello, sería preciso que este tipo de población recibiera intervención temprana o participase en programas de AT para normalizar su desarrollo en las distintas áreas (motora, comunicativa-lingüística y cognitiva), y/o ser derivados desde los servicios de cardiología infantil al alta hospitalaria.

\section{Metodología}

Este estudio de carácter cuantitativo, prospectivo y de corte transversal, que utiliza un muestreo no probabilístico, permite conocer en profundidad situaciones particulares de infantes con CC. Cuenta con un muestreo de 30 participantes, 22 varones $(73 \%)$ y 8 mujeres $(27 \%)$, en un rango de edad entre I I y 42 meses $(\overline{\mathrm{x}} 2$ I meses +4 días) cuya característica es que todos fueron intervenidos quirúrgicamente durante los primeros seis meses de vida mediante CEC, en un tiempo medio de 261,96 minutos. La edad gestacional (EG) media es de $38+$ 4 (intervalo: $35-40$ semanas) y el peso es de 3092 gr. (intervalo: 2055 gr. a 4300 gr.). La longitud media de todos los participantes corresponde a 49, I $6 \mathrm{~cm}$ (intervalo: $43 \mathrm{~cm}$ a 54 $\mathrm{cm})$, el perímetro cefálico (PC) se sitúa en 34,I 2 cm (intervalo: $29,5 \mathrm{~cm}$ a $36,5 \mathrm{~cm}$ ), el $100 \%$ de la muestra tuvo un tipo de parto único, Apgar (I’) se sitúa en $\bar{x} 7,68$ (intervalo: 4 a 9), Me: 8 y Apgar ( 5 ') en $\overline{\mathrm{x}}$ 8,56 (intervalo: 6 a Io), Me:9. 


\begin{tabular}{|c|c|}
\hline Sexo & $\begin{array}{l}22 \text { varones }(73 \%) \text { y } 8 \text { mujeres } \\
(27 \%)\end{array}$ \\
\hline$E G$ & $\begin{array}{l}38 \pm 4 \text { semanas } \\
\text { (Intervalo: } 35 \text { - } 40 \text { semanas) }\end{array}$ \\
\hline Peso medio & $\begin{array}{l}3092 \text { gr. } \\
\text { (Intervalo: } 2055 \text { gr. }-4300 \text { gr.) }\end{array}$ \\
\hline Talla & $49,1 \mathrm{~cm}$ (Intervalo: $43 \mathrm{~cm}$ a $54 \mathrm{~cm}$ ) \\
\hline PC & $\begin{array}{l}34,1 \mathrm{~cm} \\
\text { (Intervalo: } 29,5 \mathrm{~cm}-36,5 \mathrm{~cm} \text { ) }\end{array}$ \\
\hline Parto & Único (100\%) \\
\hline $\mathrm{CC}$ & $\begin{array}{l}\text { Transposición de las grandes } \\
\text { arterias (TGA, } 41 \% \text { ) Comunicación } \\
\text { interventricular (CIV, 21\%) } \\
\text { Coartación de la aorta (CoA; 6\%), } \\
\text { Canal AV (CAV, 9\%), Comunicaciór } \\
\text { Interauricular (CIA, 6\%) }\end{array}$ \\
\hline $\begin{array}{l}\text { Intervención } \\
\text { CEC }\end{array}$ & $100 \%$ de los participantes \\
\hline
\end{tabular}

Fuente: elaboración propia a partir de los datos obtenidos en $\mathrm{N}=30$.

Los participantes fueron reclutados desde las consultas de cardiología infantil del Hospital Universitario La Paz de Madrid y la Fundación Menudos Corazones ${ }^{\mathrm{I}}$. El procedimiento ha sido el de cita previa a los padres de los niños con CC y realizando una valoración con la Escala de Desarrollo Infantil Bayley-III. Previamente, los progenitores eran informados sobre esta investigación y para que sus hijos pudieran participar debían cumplimentar un formulario de consentimiento.

I. Menudos Corazones, fundación de ayuda a los niños con problemas de corazón, es una entidad sin ánimo de lucro cuyo objetivo es facilitar el desarrollo integral y la mejora de la calidad de vida de los niños y los jóvenes con cardiopatía congénita, así como de sus familiares. En este sentido, ofrece a las familias diferentes programas asistenciales para apoyarles y acompañarles cuando tienen un hijo con una cardiopatía. Desarrolla programas gratuitos, como la atención psicológica personalizada, el acompañamiento y las actividades lúdicoeducativas a los niños en hospitales, o el alojamiento para familias que han de desplazarse a Madrid por hospitalización de sus hijos.
Los objetivos de este estudio centran su foco en identificar comorbilidades ${ }^{2}$ en infantes con CC operadas al nacimiento mediante CEC, así como en diseñar directrices para su seguimiento e intervención temprana. Para desarrollar el primero, se ha evaluado el desarrollo cognitivo, motor y el área comunicativo-lingüística en los niños con CC, con el objetivo de conocer los factores de riesgo biomédicos e identificar la repercusión existente en su desarrollo y posibles dificultades en el mismo.

El análisis de datos se realizará en base a los siguientes criterios, los cuales están agrupados en dos dimensiones: la biomédica y la dimensión de neurodesarrollo.

\section{- Biomédicos}

- Datos antropométricos al nacimiento: EG, sexo, parto (múltiple/único), peso, longitud y PC.

- Antecedentes perinatales inmediatos: Score de Apgar I y II, realizado en el primer y en el quinto minuto de vida del neonato.

- Identificación del tipo de cardiopatía congénita presente en los neonatos, la cual se puede diagnosticar durante el desarrollo gestacional y/o tras el nacimiento del bebé.

- Intervención quirúrgica a través de la CEC y duración de la misma.

- Neurodesarrollo

- Motor: nivel global del desarrollo motor del niño con CC intervenido quirúrgicamente con $\mathrm{CEC}$, teniendo en cuenta el resultado total obtenido a través de las pruebas de motricidad fina y gruesa.

- Lenguaje: nivel global del desarrollo lingüístico del niño con CC, alcanzado a través de las pruebas de comunicación receptiva y expresiva.

2. El término de comorbilidad, también conocido como morbilidad asociada, se utiliza para describir dos o más enfermedades que ocurren en la misma persona (NIH, 20I7). 
- Cognitivo: nivel global del desarrollo cognitivo del impúber sometido a CEC, a través de distintas pruebas como la manipulación de objetos, exploración sensorio-motriz, etc.

Se utilizó la Escala de Desarrollo Infantil Bayley-III ${ }^{3}$ como método de evaluación del neurodesarrollo y se realizaron dos edades de cohorte entre los participantes, una de los I I a los 20 meses y otra de los 2 I a los 42 meses. Para obtener el nivel global del desarrollo motor se tuvieron en cuenta las puntuaciones compuestas obtenidas a través de las prueba de motricidad fina (FM) y de motricidad gruesa (GM). Además, con esta escala se valoró tanto el área comunicativo-lingüística, integrando la comunicación expresiva (EC) y receptiva (RC) como el área cognitiva del niño sometido a CEC.

Los datos se procesaron informáticamente mediante una base de datos en formato Microsoft Excel, que más tarde fue importada para su tratamiento estadístico en el programa SPSS versión 23.0. Se consideran estadísticamente significativos los valores de $\mathrm{p}<0,05$.

Para la descripción de variables cuantitativas continuas se utilizó la media junto con la desviación estándar, mediana y rango. Las variables cualitativas se describirán mediante frecuencias absolutas y frecuencias relativas expresadas en porcentaje.

Las comparaciones entre variables cuantitativas continuas se realizó principalmente mediante T de Student o ANOVA o, alternativamente, pruebas no-paramétricas Kruskal-Wallis o U de Mann-Whitney cuando fue necesario. El análisis de frecuencias entre variables cualitativas se realizó mediante la prueba de la $\mathrm{c}^{2} \mathrm{o}$ el test exacto de Fisher. La correlación entre variables cuantitativas se midió mediante el coeficiente de correlación de Pearson.

3. Destinada a infantes de uno a cuarenta y dos meses, de aplicación individual en un tiempo aproximado de cincuenta (< 2 meses) a noventa (> I 2 meses) minutos, dependiendo de la edad del evaluado.

\section{Resultados}

Todos los participantes en el estudio presentan distintos tipos de cardiopatías como CIA, CIV, CoA, CAV, TGA, cor triatriatum, ductus arterioso, Insuficiencia Triscupídea Severa (ITS) y Transposición Corregida de las Grandes Arterias (LTGA). Estos resultados coinciden con la prevalencia observada en la población con CC con los siguientes porcentajes: TGA (4I \%), CIV (2I \%), CoA (6\%) y CAV (9\%), ya que las restantes son diagnósticos secundarios dentro de las relevantes. El total de la muestra recogida del Hospital Universitario La Paz de Madrid y la Fundación Menudos Corazones nos indica que la CC de TGA se manifiesta con mayor prevalencia (4I \%), seguida de CIV (2I \%) para esta etapa.

\section{Gráfico 1. Tipología CC en el Hospital Universitario La Paz de Madrid y la Fundación Menudos Corazones}

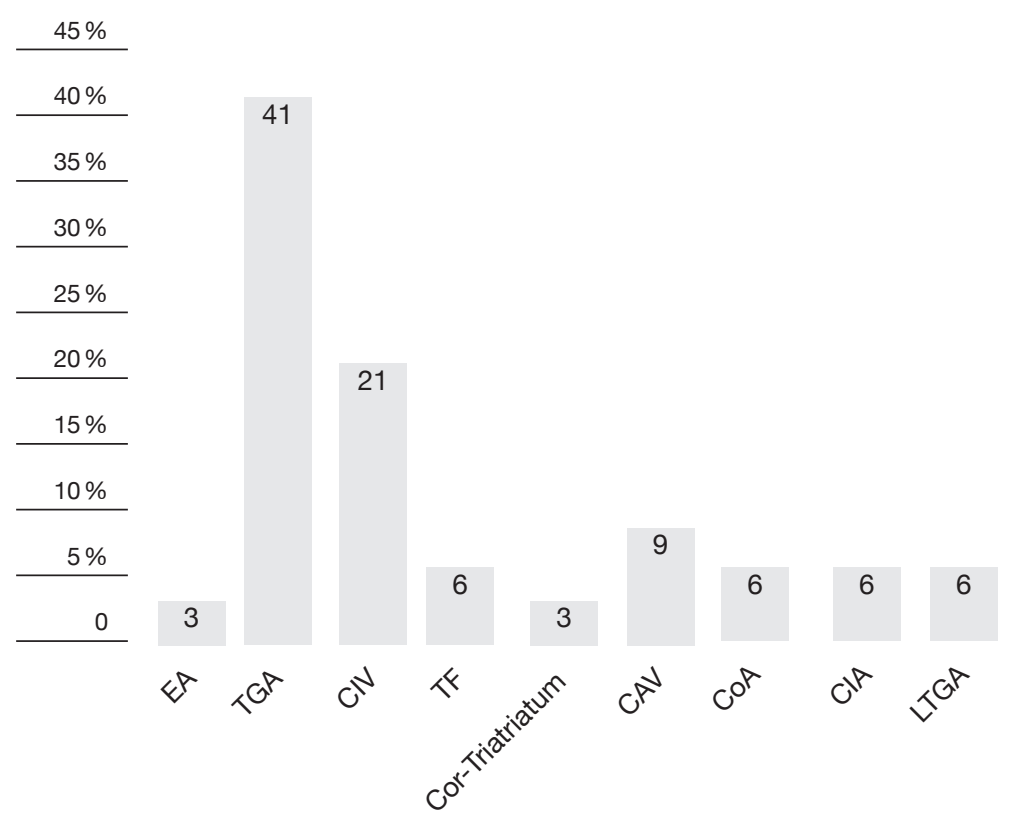

Fuente: elaboración propia a partir del tipo de CC recogida de $\mathrm{N}=30$ en el Hospital Universitario La Paz de Madrid y la Fundación Menudos Corazones. 
Fruto de este estudio se obtienen diversas puntuaciones compuestas que emergen de las áreas del desarrollo valoradas, a través de la Escala de Desarrollo Bayley-III, en los menores con anomalías cardiacas. La Tabla 2 muestra estos datos.

Los resultados de las puntaciones compuestas obtenidos en cada área de desarrollo de los 30 participantes evidencian tendencias para estas edades en lentificación en el área comunicativa-lingüística $(\overline{\mathrm{x}} 87, \mathrm{I})$. Dittrich et al. (2003) defendían que cuando se realizaba una intervención quirúrgica en el período neonatal se producían secuelas en el habla y en el lenguaje.

Los resultados también nos muestran que existen morbilidades en el área motora $(\overline{\mathrm{x}} 89,2)$. Autores como Mulkey et al. (20I4) indican que los infantes con CC, intervenidos

Tabla 2. Puntuaciones de las áreas del desarrollo de cada sujeto

\begin{tabular}{|c|c|c|c|c|c|c|c|c|}
\hline & \multicolumn{2}{|c|}{ Cognitivo } & \multicolumn{3}{|c|}{ Lenguaje } & \multicolumn{3}{|c|}{ Motor } \\
\hline SJ & & Compuesta & Receptivo & Expresivo & Compuesta & Fino & Grueso & Compuesta \\
\hline 1 & 47 & 75 & 14 & 11 & 59 & 35 & 46 & 82 \\
\hline 2 & 83 & 140 & 35 & 38 & 106 & 48 & 63 & 110 \\
\hline 3 & 32 & 60 & 9 & 6 & 53 & 18 & 19 & 46 \\
\hline 4 & 46 & 80 & 17 & 10 & 71 & 24 & 48 & 67 \\
\hline 5 & 81 & 105 & 29 & 43 & 91 & 59 & 64 & 127 \\
\hline 6 & 74 & 110 & 29 & 26 & 89 & 41 & 54 & 107 \\
\hline 7 & 34 & 60 & 10 & 9 & 59 & 39 & 45 & 110 \\
\hline 8 & 73 & 110 & 32 & 28 & 97 & 41 & 43 & 73 \\
\hline 9 & 76 & 100 & 22 & 23 & 68 & 39 & 56 & 70 \\
\hline 10 & 74 & 110 & 27 & 30 & 89 & 54 & 56 & 121 \\
\hline 11 & 77 & 105 & 27 & 29 & 83 & 46 & 58 & 94 \\
\hline 12 & 38 & 95 & 14 & 14 & 97 & 28 & 30 & 76 \\
\hline 13 & 49 & 95 & 16 & 16 & 83 & 33 & 49 & 97 \\
\hline 14 & 75 & 95 & 34 & 39 & 94 & 47 & 62 & 88 \\
\hline 15 & 35 & 85 & 15 & 14 & 103 & 26 & 37 & 85 \\
\hline 16 & 42 & 105 & 15 & 15 & 103 & 28 & 39 & 91 \\
\hline 17 & 37 & 75 & 11 & 10 & 59 & 23 & 34 & 49 \\
\hline 18 & 65 & 105 & 21 & 19 & 77 & 38 & 53 & 94 \\
\hline 19 & 45 & 95 & 16 & 17 & 94 & 29 & 40 & 83 \\
\hline 20 & 27 & 60 & 6 & 4 & 47 & 16 & 20 & 46 \\
\hline 21 & 48 & 90 & 18 & 20 & 91 & 31 & 46 & 82 \\
\hline 22 & 45 & 95 & 16 & 15 & 91 & 30 & 46 & 94 \\
\hline 23 & 49 & 105 & 17 & 17 & 97 & 31 & 46 & 82 \\
\hline 24 & 44 & 90 & 15 & 15 & 83 & 30 & 40 & 79 \\
\hline 25 & 62 & 105 & 25 & 29 & 103 & 40 & 58 & 115 \\
\hline 26 & 59 & 90 & 24 & 22 & 83 & 38 & 55 & 94 \\
\hline 27 & 53 & 95 & 21 & 19 & 89 & 35 & 51 & 97 \\
\hline 28 & 9 & 100 & 8 & 8 & 127 & 6 & 8 & 100 \\
\hline 29 & 40 & 105 & 15 & 14 & 109 & 29 & 42 & 110 \\
\hline 30 & 13 & 115 & 35 & 32 & 118 & 45 & 60 & 107 \\
\hline
\end{tabular}

Fuente: elaboración propia a partir de los resultados obtenidos por cada niño en las áreas del desarrollo, valorados con la Escala de Desarrollo Infantil Bayley-III. 
quirúrgicamente, muestran un desarrollo motor inferior tanto grueso como fino. En ambas áreas de desarrollo, lenguaje y motora, se evidencia un desarrollo categorizado en normal-lento.

A continuación, se han analizado las puntuaciones medias obtenidas en los dos grupos etarios, divididos en dos grupos de I I a 20 meses y de 2 I a 42 meses. Como se observa, entre ambos grupos de edad se evidencian diferencias en las puntuaciones obtenidas en el área cognitiva $(\overline{\mathrm{x}} 88,3$ y $\overline{\mathrm{x}}$ I04, I; P $=$ I 6 y P $=63$ respectivamente) y en el desarrollo motor ( $\overline{\mathrm{x}}$ 82,5 y $\overline{\mathrm{x}}$ 97,9; $\mathrm{P}=\mathrm{I} 2$ y $\mathrm{P}=42$ respectivamente). Sin embargo, las puntuaciones que se obtienen en el área comunicativo-lingüística en los dos grupos etarios son bastante similares ( $\overline{\mathrm{x}} 86$ y $\overline{\mathrm{x}} 86,5 ; \mathrm{P}=\mathrm{I} 8$ respectivamente), observándose una ausencia de progreso en la misma.

\section{Gráfico 2. Puntuaciones obtenidas en el desarrollo motor, del lenguaje y cognitivo}

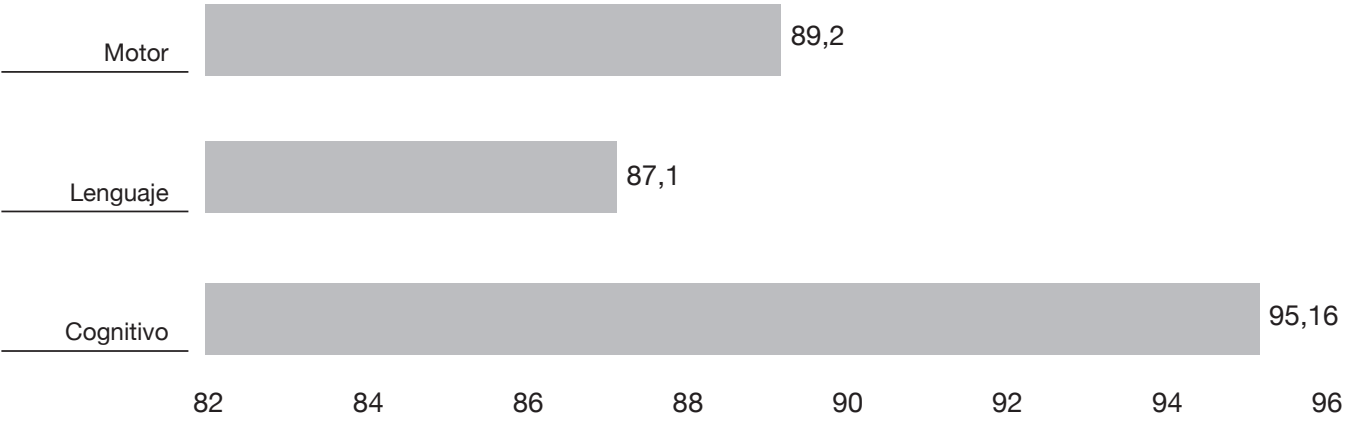

Fuente: elaboración propia a partir de las puntuaciones medias obtenidas en la valoración de las tres áreas del desarrollo (motor, comunicativa-lingüística, lenguaje) a $\mathrm{N}=30$.

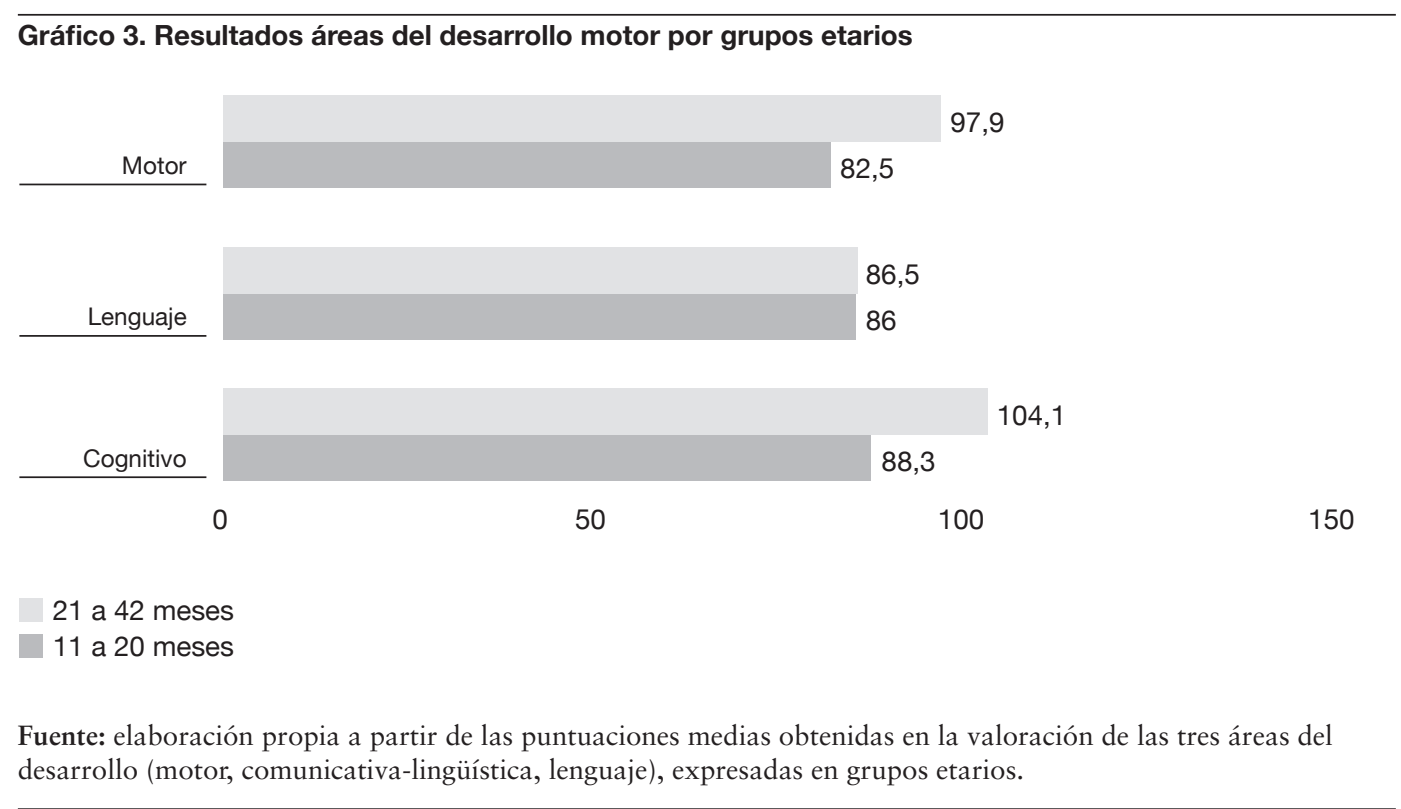


Comparando la edad de valoración de los infantes con los resultados obtenidos en la Escala de Desarrollo Infantil Bayley-III, se puede afirmar que este tipo de población muestra una tendencia a presentar un desarrollo normallento.

\begin{tabular}{|c|c|c|}
\hline \multicolumn{2}{|c|}{$\begin{array}{l}\text { Correlación desarrollo y edad de } \\
\text { valoración }\end{array}$} & \multirow{2}{*}{$\begin{array}{c}\text { EDAD } \\
1\end{array}$} \\
\hline \multirow{3}{*}{ EDAD } & Pearson Correlation & \\
\hline & Sig. (2-tailed) & \\
\hline & $\mathrm{N}$ & 30 \\
\hline \multirow{3}{*}{ Cognitivo } & Pearson Correlation &, 796 \\
\hline & Sig. (2-tailed) &, 000 \\
\hline & $\mathrm{N}$ & 30 \\
\hline \multirow{3}{*}{$\begin{array}{l}\text { Cognitivo } \\
\text { Compuesto }\end{array}$} & Pearson Correlation & ,413 \\
\hline & Sig. (2-tailed) & ,023 \\
\hline & $N$ & 30 \\
\hline \multirow{3}{*}{ Lenguaje Receptivo } & Pearson Correlation &, 727 \\
\hline & Sig. (2-tailed) &, 000 \\
\hline & $\mathrm{N}$ & 30 \\
\hline \multirow{3}{*}{ Lenguaje Expresivo } & Pearson Correlation &, 781 \\
\hline & Sig. (2-tailed) &, 000 \\
\hline & $\mathrm{N}$ & 30 \\
\hline \multirow{3}{*}{$\begin{array}{l}\text { Lenguaje } \\
\text { Compuesto }\end{array}$} & Pearson Correlation &, 015 \\
\hline & Sig. (2-tailed) & ,936 \\
\hline & $N$ & 30 \\
\hline \multirow{3}{*}{ Motor Fino } & Pearson Correlation & ,707 \\
\hline & Sig. (2-tailed) &, 000 \\
\hline & $\mathrm{N}$ & 30 \\
\hline \multirow{3}{*}{ Motor Grueso } & Pearson Correlation & ,633 \\
\hline & Sig. (2-tailed) &, 000 \\
\hline & $\mathrm{N}$ & 30 \\
\hline \multirow{3}{*}{ Motor Compuesto } & Pearson Correlation & ,304 \\
\hline & Sig. (2-tailed) & ,103 \\
\hline & $N$ & 30 \\
\hline
\end{tabular}

Fuente: elaboración propia a partir de la edad de valoración de los infantes y los resultados obtenidos en las áreas del desarrollo, evidenciando morbilidades en el total de la muestra.
Posteriormente, se ha realizado la media por edades en rangos de doce meses (hasta I 2, 24, 36 y 42). Con los resultados obtenidos podemos concluir que los 12 meses de edad cronológica marcan un punto de inflexión para la derivación a servicios de AT, ya que a los 24 meses los datos evidencian la existencia de lentificaciones en el desarrollo.

Una vez más, observamos que tanto el desarrollo motor como el del lenguaje son inferiores a la edad estimada.

A su vez, se observa como el desarrollo motor es inferior hasta los I 2 meses $(8 \mathrm{I}, 6)$ y va aumentando progresivamente hasta los 24 meses $(85,3)$, luego hasta los 36 meses $(88,5)$ $y$, finalmente, hasta los 42 meses $(89,2)$. No obstante, aunque el desarrollo del área motora se va normalizando con el paso del tiempo, los resultados que se obtienen de los participantes de este estudio, valorados una única vez, continúan categorizándose normal-lento cuando alcanzan la edad de 42 meses.

Se observa que existe retraso en el área comunicativo-lingüística debido a la disminución en la puntuación de esta área que se evidencian entre los I 2 y los 24 meses de edad $(86,3)$, cuando el infante debería comenzar a emitir sus primeras palabras, prolongándose hasta los 42 meses $(87, \mathrm{I})$. Sin embargo, a nivel general, los participantes muestran un desarrollo normal $(9 \mathrm{I}, 8)$ hasta los doce meses.

A nivel cognitivo se muestra un avance progresivo hasta los 42 meses. Pero su desarrollo en esta área es el mismo desde los o hasta los 24 meses, obteniendo la misma puntuación. Por ello, se abre una futura línea de investigación para identificar los posibles factores que intervienen en el retraso del desarrollo que se evidencia a partir de los datos obtenidos. Autores como Schaefer et al. (2013) apuntan que debido a estas alteraciones, los infantes con CC sometidos a CEC obtienen peores resultados académicos.

Comparando las variables biomédicas con los resultados de la Escala de Desarrollo Bayley- 


\section{Gráfico 4. Edad con mayores morbilidades}

$\begin{array}{r}100 \\ \hline 95 \\ \hline 90 \\ \hline 85 \\ \hline 80 \\ \hline 75 \\ \hline 70 \\ \hline\end{array}$
Hasta 12 meses
Hasta 24 meses
Hasta 36 meses
Hasta 42 meses

Cognitivo

Lenguaje

Motor

Fuente: elaboración propia a partir de las puntuaciones medias obtenidas en las áreas del desarrollo correspondientes a rangos de doce meses, estimando el punto de inflexión para la derivación a Servicios de AT.

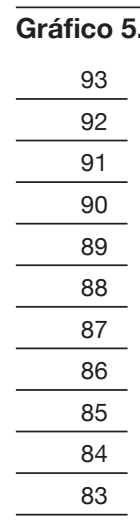

Fuente: elaboración propia a partir de las puntuaciones medias obtenidas de $\mathrm{N}=30$ en el área del lenguaje, observando un desarrollo inferior a lo esperado por edad cronológica a partir de los doce meses.

III, se comprueba que no existen diferencias significativas entre las diferentes áreas de desarrollo. Por ello, se afirma que hay ausencia de indicadores biomédicos que inciden en el desarrollo, aunque se tiene que tener en cuenta el riesgo ambiental que se inicia tras la intervención quirúrgica, pues las variables biomédicas no son significativas. Por lo que la AT tendría un carácter preventivo. 


\begin{tabular}{|c|c|c|}
\hline \multicolumn{2}{|c|}{ Correlación desarrollo y EG } & \multirow{2}{*}{$\begin{array}{c}\text { EG } \\
1 \\
\end{array}$} \\
\hline \multirow{3}{*}{$E G$} & Pearson Correlation & \\
\hline & Sig. (2-tailed) & \\
\hline & $\mathrm{N}$ & 28 \\
\hline \multirow{3}{*}{ Cognitivo } & Pearson Correlation &, 138 \\
\hline & Sig. (2-tailed) & ,483 \\
\hline & $\mathrm{N}$ & 28 \\
\hline \multirow{3}{*}{$\begin{array}{l}\text { Cognitivo } \\
\text { Compuesto }\end{array}$} & Pearson Correlation & ,064 \\
\hline & Sig. (2-tailed) &, 747 \\
\hline & $\mathrm{N}$ & 28 \\
\hline \multirow{3}{*}{\begin{tabular}{|l} 
Lenguaje \\
Receptivo
\end{tabular}} & Pearson Correlation & ,038 \\
\hline & Sig. (2-tailed) & ,847 \\
\hline & $\mathrm{N}$ & 28 \\
\hline \multirow{3}{*}{$\begin{array}{l}\text { Lenguaje } \\
\text { Expresivo }\end{array}$} & Pearson Correlation & ,032 \\
\hline & Sig. (2-tailed) & ,873 \\
\hline & $\mathrm{N}$ & 28 \\
\hline \multirow{3}{*}{$\begin{array}{l}\text { Lenguaje } \\
\text { Compuesto }\end{array}$} & Pearson Correlation & 015 \\
\hline & Sig. (2-tailed) &, 940 \\
\hline & $\mathrm{N}$ & 28 \\
\hline \multirow{3}{*}{ Motor Fino } & Pearson Correlation &, 122 \\
\hline & Sig. (2-tailed) &, 537 \\
\hline & $\mathrm{N}$ & 28 \\
\hline \multirow{3}{*}{$\begin{array}{l}\text { Motor } \\
\text { Grueso }\end{array}$} & Pearson Correlation & ,066 \\
\hline & Sig. (2-tailed) &, 739 \\
\hline & $\mathrm{N}$ & 28 \\
\hline \multirow{3}{*}{$\begin{array}{l}\text { Motor } \\
\text { Compuesto }\end{array}$} & Pearson Correlation & 087 \\
\hline & Sig. (2-tailed) & ,659 \\
\hline & $N$ & 28 \\
\hline
\end{tabular}

Fuente: elaboración propia a partir de la comparación de las variable biomédica EG con los resultados obtenidos en el desarrollo, afirmando ausencia de indicadores biomédicos incidentes en el desarrollo.

Teniendo en cuenta los resultados, podemos concluir que se cumple el objetivo del estudio que pretendía identificar morbilidades en infantes con CC operadas al nacimiento mediante CEC.

En consecuencia, es preciso protocolizar y diseñar directrices para esta población con CC y derivar a programas interdisciplinares de seguimiento e intervención temprana, alcanzando el segundo objetivo de esta

\begin{tabular}{|c|c|c|}
\hline \multicolumn{3}{|c|}{ Tabla 5. Correlación desarrollo y duración CEC } \\
\hline \multicolumn{2}{|c|}{ Correlación desarrolloy duración CEC } & \multirow{2}{*}{\begin{tabular}{|c|} 
TIEMPO \\
1 \\
\end{tabular}} \\
\hline \multirow{3}{*}{ TIEMPO } & Pearson Correlation & \\
\hline & Sig. (2-tailed) & \\
\hline & $\mathrm{N}$ & 23 \\
\hline \multirow{3}{*}{ Cognitivo } & Pearson Correlation & ,186 \\
\hline & Sig. (2-tailed) & ,397 \\
\hline & $\mathrm{N}$ & 23 \\
\hline \multirow{3}{*}{$\begin{array}{l}\text { Cognitivo } \\
\text { Compuesto }\end{array}$} & Pearson Correlation &,- 088 \\
\hline & Sig. (2-tailed) & ,689 \\
\hline & $\mathrm{N}$ & 23 \\
\hline \multirow{3}{*}{$\begin{array}{l}\text { Lenguaje } \\
\text { Receptivo }\end{array}$} & Pearson Correlation & ,219 \\
\hline & Sig. (2-tailed) & ,315 \\
\hline & $\mathrm{N}$ & 23 \\
\hline \multirow{3}{*}{$\begin{array}{l}\text { Lenguaje } \\
\text { Expresivo }\end{array}$} & Pearson Correlation & ,292 \\
\hline & Sig. (2-tailed) & ,176 \\
\hline & $\mathrm{N}$ & 23 \\
\hline \multirow{3}{*}{$\begin{array}{l}\text { Lenguaje } \\
\text { Compuesto }\end{array}$} & Pearson Correlation & ,063 \\
\hline & Sig. (2-tailed) &, 776 \\
\hline & $\mathrm{N}$ & 23 \\
\hline \multirow{3}{*}{ Motor Fino } & Pearson Correlation &, 161 \\
\hline & Sig. (2-tailed) & ,464 \\
\hline & $\mathrm{N}$ & 23 \\
\hline \multirow{3}{*}{$\begin{array}{l}\text { Motor } \\
\text { Grueso }\end{array}$} & Pearson Correlation & ,081 \\
\hline & Sig. (2-tailed) &, 712 \\
\hline & $\mathrm{N}$ & 23 \\
\hline \multirow{3}{*}{$\begin{array}{l}\text { Motor } \\
\text { Compuesto }\end{array}$} & Pearson Correlation &,- 113 \\
\hline & Sig. (2-tailed) & ,606 \\
\hline & $\mathrm{N}$ & 23 \\
\hline
\end{tabular}

Fuente: elaboración propia a partir de la comparación de la variable biomédica tiempo de intervención de CEC, afirmando ausencia de indicadores biomédicos incidentes en el desarrollo.

investigación porque, en la actualidad, en la legislación presente de la Comunidad de Madrid no consta ninguna normativa que regule la AT que deberían recibir estos niños. A su vez, se considera que es necesaria la creación de normativas reguladas que estén ligadas a la atención de este tipo de población con la finalidad de normalizar su desarrollo durante los primeros meses de edad, especialmente en aquellas áreas en las que el desarrollo es inferior. 
Lógicamente, no todos los sujetos con CC presentaran retardo en el desarrollo aunque estamos hablando de una predisposición a presentarlo, teniendo la necesidad de recibir AT.

\section{Conclusiones}

Las valoraciones realizadas nos indican que la mayoría de los menores presentan lentificaciones en el desarrollo motor y en el área comunicativo-lingüística. El desarrollo en el área motora se va normalizando según va creciendo el niño, aunque continua mostrándose inferior a lo esperado por su edad cronológica.

Sin embargo, el desarrollo del lenguaje disminuye cuando el niño crece, manteniéndose así hasta los 42 meses de edad cronológica (edad máxima valorada en el estudio). Al principio, el desarrollo del lenguaje se sitúa en rangos normales de desarrollo porque el lenguaje todavía no está presente, pero cuando éste debe aparecer (aproximadamente a los I 2 meses), esta área muestra un desarrollo inferior a lo esperado por la edad cronológica.

Centrándonos en las variables biomédicas, se indica que no se han encontrado influencias en el desarrollo, aunque se evidencian similitudes entre aquellos participantes que muestran morbilidades en el desarrollo.

Teniendo en cuenta todo lo anterior, se perfila como favorable que los niños que presentan CC reciban o puedan ser derivados a programas de AT para normalizar su desarrollo en los dos primeros años de vida y es necesario diseñar protocolos interdisciplinares de detección, despistaje e intervención desde un modelo centrado en la familia para este tipo de población.

Este modelo tiene que estar centrado en los siguientes términos:

- Evaluación inicial en desarrollo madurativo a partir del primer mes de vida.

- Elaboración de informes psicopedagógicos, con dictamen de necesidades educativas para tratamiento de AT.

- Diseñar programas para normalizar el desarrollo del infante con CC intervenido quirúrgicamente tras su nacimiento. En primer lugar, se realizará una entrevista a los progenitores para que nos aporten datos biológicos, psicológicos... sobre el menor. Seguidamente, se realizará una evaluación inicial de las áreas de desarrollo y se elaborará un informe dictaminando qué áreas precisan AT.

Teniendo en cuenta a cada sujeto de manera individualizada, se establecerán unos objetivos y, con ellos, se propondrán actividades que permitan alcanzarlos, normalizando el desarrollo del infante.

- Incorporación de profesionales cualificados del ámbito psicopedagógico y de la salud en los programas de seguimiento para esta población.

Por último, como línea futura de investigación, sería conveniente realizar más valoraciones a partir de los 36 meses para asegurar un desarrollo adecuado al esperado por edad, porque parece existir un estancamiento en el mismo. 


\section{Referencias bibliográficas}

Aguirre, P. et al. (2008): Manual de atención al alumnado con necesidades especificas de apoyo educativo por padecer enfermedades raras y crónicas (en línea). <http://www. juntadeandalucia.es/educacion/webportal/ ishare-servlet/content/ca I 7 bcc7-9 I 46-45 5 ba23d-7cdea9ae6546>, acceso 3 de septiembre de 2016.

Alcover, E. et al. (I999): “Seguimiento neurológico y somatométrico de operados cardíacos antes de los 45 días de vida”. Anales Españoles de Pediatría, 5I (I): 60-63.

Armele, M. et al. (2014): "Intervención neuropsicológica en un caso de trastorno del desarrollo generado por una cardiopatía congénita”. Revista Chilena de Neuropsicología, 9 (2): 80-84.

Arretz, C. (2000): "Cirugía de las cardiopatías congénitas en el recién nacido y lactante”. Revista Chilena de Pediatría, 7I (2).

Azcoaga, J. E. (I979): Aprendizaje fisiológico y aprendizaje pedagógico. Buenos Aires: Paidós.

Bayley, N. (2005): Bayley-III, Escalas de desarrollo infantil-III (en línea). <http://www. pearsonclinical.es/producto/I 2o/bayley-iiiescalas-bayley-de-desarrollo-infantil-iii>, acceso 24 de mayo de 2017.

Dittrich, H. et al. (2003): "Neurodevelopment at I year of age in infants with congenital heart disease". Heart, 89: 436-44I.

DrugFacts (2017): NIH National Institute on Drug abuse. Advancing Addiction Science (en línea). <https://www.drugabuse.gov/es/publicaciones/ drugfacts/la-comorbilidad-entre-la-adiccion-yotros-trastornos-mentales $>$, acceso 24 de mayo de 2017.

Esquivel, F. et al. (2013): “Alteraciones electroencefalográficas y del neurodesarrollo en niños portadores de cardiopatías congénitas severas” (estudio preliminar). Gaceta Médica de México, I49: 605-6I2.
GAT (2000): Libro Blanco de la Atención Temprana. Madrid: Real Patronato sobre Discapacidad (en línea). <http://www. juntadeandalucia.es/salud/servicios/ contenidos/andaluciaessalud/doc/ LibroBlancoAtenci,'nTemprana.pdf>, acceso I de marzo de 2017

González, S. (20I3): Atención Temprana en niños y niñas con TEA (Trabajo Final de Máster). Instituto Superior de Psicología (ISEP), España.

Gunn, J. et al. (2OI2): "Perioperative amplitudeintegrated EEG and neurodevelopment in infants with congenital heart disease". Intensive Care Med, 38: I 539-I 547.

Magliola, R. et al. (2009): "Cirugía cardíaca reparadora en recién nacidos. Experiencia de 5 años en cirugía neonatal con circulación extracorpórea”. Arch Argent Pediatr, IO7 (5): 417-422.

Matsuzaki, T. et al. (20I0): "Neurodevelopment in I- year-old Japanese infants after congenital heart surgery”. Pedriatrics Intenational, 52: $420-427$.

Mulkey, S. et al. (20I4): “Academic proficiency in children after early congenital heart disease surgery". Pediatric Cardiol, 35: 344-352.

NIH (20I7): National Institute on Drug Abuse (en línea). <https://vsearch.nlm.nih.gov/vivisimo/ cgi-bin/query-meta? $\%{ }_{3}$ Aproject=medlineplusspanish\&v\% 3 Asources=medlineplus-spanishbundle\&query=comorbilidad $+>$, acceso el 5 de marzo de 2017.

Organización Mundial de la Salud (2016): Enfermedades Crónicas (en línea). <http:// www.who.int/topics/chronic_diseases/es/>, acceso 3 de septiembre de 2016 .

Schaefer, C. et al. (2013): "Neurodevelopmental outcome, psychological adjustment and quality of life in adolescents with congenital heart disease". Developmental Medicine \& Child Neurology, 55: I I 43-I I 49. 
Villagrá, F. (20I4): Qué son las cardiopatías congénitas (en línea). <http://www.

menudoscorazones.org/cardiopatias-congenitas/ que-son-las- cardiopatias-congenitas/>, acceso 6 de abril de 2015 .

Williams, I. et al. (20I 2a): "The association of fetal cerebrovascular resistance with early neurodevelopment in single ventricle congenital heart disease". American Heart Journal, I 65

(4): 544-550.

Williams, I. et al. (2012b): "Fetal cerebrovascular resistance and neonatal EEG predict I 8-month neurodevelopmental outcome in infants with congenital heart disease". Ultrasound Obstest Gynecol, 40: 304-309. 